\title{
Comparison of Topography- and Aperture-Dependent Motion Compensation Algorithms for Airborne SAR
}

\author{
Pau Prats, Member, IEEE, Karlus A. Câmara de Macedo, Andreas Reigber, Member, IEEE, \\ Rolf Scheiber, and Jordi J. Mallorqui, Member, IEEE
}

\begin{abstract}
This letter presents a comparison between three Fourier-based motion compensation (MoCo) algorithms for airborne synthetic aperture radar (SAR) systems. These algorithms circumvent the limitations of conventional MoCo, namely the assumption of a reference height and the beam-center approximation. All these approaches rely on the inherent time-frequency relation in SAR systems but exploit it differently, with the consequent differences in accuracy and computational burden. After a brief overview of the three approaches, the performance of each algorithm is analyzed with respect to azimuthal topography accommodation, angle accommodation, and maximum frequency of track deviations with which the algorithm can cope. Also, an analysis on the computational complexity is presented. Quantitative results are shown using real data acquired by the Experimental SAR system of the German Aerospace Center (DLR).
\end{abstract}

Index Terms-Calibration, image registration, interferometry, motion compensation (MoCo), synthetic aperture radar (SAR).

\section{INTRODUCTION}

$\mathbf{M}$ OTION compensation (MoCo) represents a key issue in the focusing of airborne synthetic aperture radar (SAR) data. The fact that the platform does not follow an ideally linear trajectory turns into a severe degradation of the impulse response function (IRF) if the deviations are not considered. Hence, the SAR focusing operation must ensure a proper correction of such deviations by means of a modified processing scheme. Moreira et al. [1] proposed the two-step MoCo approach integrated in the extended chirp scaling (ECS) algorithm, which allows the retrieval of accurate interferometric products with airborne systems. This two-step MoCo consists of the application of a phase and envelope correction in the beginning of the processing (first-order $\mathrm{MoCo}$ ), where the deviations are corrected for a reference range and height. A range-dependent correction is not possible at this stage since range cell migration (RCM) is still present in the data. A second

Manuscript received September 29, 2006; revised January 29, 2007. This work was supported in part by the Spanish MCYT and FEDER funds under Project TEC2005-06863-C02-01. The work of K. A. Câmara de Macedo is supported by a grant from CAPES, Brazil.

P. Prats, K. A. Câmara de Macedo, and R. Scheiber are with the Microwaves and Radar Institute, Deutsche Zentrum für Luft- und Raumfahrt, 82234 Oberpfaffenhofen, Germany (e-mail: pau.prats@dlr.de; karlus.macedo@dlr.de; rolf.scheiber@dlr.de).

A. Reigber is with the Berlin University of Technology, 10587 Berlin, Germany (e-mail: anderl@cs.tu-berlin.de).

J. J. Mallorqui is with the Universitat Politècnica de Catalunya, 08034 Barcelona, Spain (e-mail: mallorqui @tsc.upc.edu).

Digital Object Identifier 10.1109/LGRS.2007.895712 range-dependent phase correction is applied after range compression and RCM correction (RCMC), and before azimuth compression (second-order MoCo). In this step, topography variations can be partially considered using an external digital elevation model (DEM), but the correction to be applied also depends on the position of the target within the beam. Commonly, a reference height is assumed for the whole scene, while the correction is applied for the center of the beam. In [2], a detailed analysis of this latter approximation is presented.

Three algorithms that circumvent these two limitations have been proposed in the literature [3]-[6]. They all rely on the time-frequency relation of the azimuth SAR signal but exploit it in different ways. Also, they use an external DEM to compute the proper phase delays to be applied to each target to correctly accommodate topography variations. The goal of this letter is to analyze the performance of each algorithm based on the accuracy in the accommodation of the different parameters of interest: topography, azimuth angle, and frequency of track deviations. Furthermore, a quantitative analysis is carried out by comparison with a time-domain SAR processor, which allows applying MoCo with no approximation.

Section II starts with a review of the limitations in conventional MoCo when applied inside Fourier-based processors. Section III revisits the three algorithms under study by presenting their main characteristics, which will allow a proper analysis and comparison of the results. Section IV makes a statement regarding the phase content of an interferometric pair when processing using the presented approaches. Section V shows the results with real data acquired by the Experimental SAR (E-SAR) system of the German Aerospace Center (DLR). Finally, Section VI brings the conclusions.

\section{Limitations in Conventional MoCo}

It is well known that the conventional two-step MoCo makes two approximations [3].

1) Topography approximation: A reference height for the whole scene is assumed to apply MoCo, which yields IRF degradation for targets at a height different than the reference one. An external DEM can be used in second-order MoCo, but this approach cannot accurately accommodate topography if it varies significantly along the synthetic aperture, which, depending on resolution and wavelength, can be as large as several hundred meters. 
2) Beam-center approximation: The correction also depends on the relative position of the target with respect to the sensor, i.e., the correction depends on the azimuthal aperture/angle. Usually, the correction is applied at the center of the beam, which results in IRF degradation when the processed azimuthal beamwidth is large. This degradation has more impact in low frequency systems, where a wide aperture is necessary to achieve a high azimuthal resolution [7].

The effects of both approximations are similar and well established in the literature [2], [3]. These are mainly a shift of the IRF, defocusing, and phase aberrations. These will turn into significant phase and coregistration errors in the generated interferogram. Note that in interferometric single-pass systems, the deviations are correlated in master and slave images; hence, the errors tend to cancel out after interferogram generation. However, interferometric repeat-pass products are usually affected by these approximations. Normally, the first of the commented approximations is the one that has more impact, but for high-precision SAR focusing, both approximations must be considered.

\section{TOPOGRAPHY AND APERTURE COMPENSATION}

To apply a topography- and aperture-dependent MoCo, the algorithms rely on the well-known time-frequency relation of the azimuth SAR signal. The mapping between time and frequency is expressed by

$$
f_{a}\left(t ; r_{0}, t_{0}\right)=\frac{2 v}{\lambda} \sin \beta=\frac{2 v^{2}}{\lambda} \frac{t_{0}-t}{\sqrt{r_{0}^{2}+v^{2} \cdot\left(t-t_{0}\right)^{2}}}
$$

where $t$ is the azimuth time, $\beta$ is the azimuth angle corresponding to an azimuth frequency $f_{a}, v$ is the forward velocity of the platform, $t_{0}$ is the zero-Doppler time, $r_{0}$ is the closest approach distance, which corresponds to $t_{0}$, and $\lambda$ is the used wavelength. The correction to be applied is both azimuth time dependent and azimuth angle/frequency dependent. An efficient solution to this problem can be achieved via block processing, which becomes the tool used by the three approaches presented next.

\section{A. Precise Topography- and Aperture-Dependent (PTA) Algorithm}

The PTA algorithm was presented in [3]. It is applied with the image already focused so that the residual phase correction is computed for each target considering its true topographic height and the real distance at each time instant, hence also accommodating the azimuth angle variation. The correction is applied in the frequency domain using the time-frequency mapping indicated by (1), in principle just by reversing the correction vector computed in the time domain. The implemented approach makes only one forward Fourier transform (FT) per block, taking a $50 \%$ overlap $(\alpha=0.5)$ to avoid discontinuities at block borders. An inverse FT is needed for each target after the correction, which results in a relatively high computational burden. (Since only one sample after the inverse FT is kept, the direct computation of that single value can save some time.) For
TABLE I

Properties of THE Algorithms Under StUdy With RESPECT TO TOPOGRAPHY, ANGLE, AND RESIDUAL ERRORS ACCOMMODATION

\begin{tabular}{lccc}
\hline Algorithm & $\delta x[\mathrm{~m}]$ & $\delta \theta_{\mathrm{az}}[\mathrm{rad}]$ & $f_{\max }^{\mathrm{res}}[\mathrm{Hz}]$ \\
\hline PTA & $v / P R F$ & $\theta_{\mathrm{az}} / N$ & $N /\left(2 L_{\mathrm{sa}}\right)$ \\
SATA & $r \theta_{\mathrm{az}} / N$ & $\theta_{\mathrm{az}} / N$ & $P R F /(2 N)$ \\
FD & $r \theta_{\mathrm{az}} N / N_{\mathrm{az}}$ & $\theta_{\mathrm{az}} N / N_{\mathrm{az}}$ & $P R F / 2$ \\
\hline
\end{tabular}

efficiency purposes, the block length should be much smaller than the synthetic aperture, e.g., 32 or 64 azimuth samples. Also, higher efficiency can be gained by changing adaptively the block size depending on track and topography variations.

The main advantage of this algorithm lies in the fact that it can accommodate the scene topography within the sample spacing. On the other hand, given that the correction is downsampled to the length of the block, the ability to accommodate both the angle and the high-frequency component of the residual error is reduced. These drawbacks can be circumvented by increasing the block size $N$. However, this increases the computational burden, hence showing a clear tradeoff between accuracy and efficiency.

\section{B. Subaperture Topography- and Aperture-Dependent (SATA) Algorithm}

The SATA algorithm was first presented in [4]. Again, accurate MoCo can be obtained by exploiting the time-frequency relation in SAR. The main difference with respect to PTA is that in this case the correction is applied before azimuth compression. The solution proposed in [4] is to use the short-time FT in blocks (or subapertures) along the azimuthal direction. The block size $N$ is a compromise between topography/angle accommodation and trajectory deviation accommodation. A larger block size increases the accuracy of the topography/angle accommodation. However, since the correction to be applied considers the track deviation in the middle of the block, the accommodation of fast track variations is reduced in this case. A small block size performs in the opposite way. A clear advantage of this algorithm is that it is computationally very efficient; the number of necessary FTs is drastically reduced when compared to the other two algorithms.

It should be noted that the accommodation of the topography depends on range (see Table I); hence, a better resolution is achieved at near range than at far range. A straightforward solution to this problem is to increase the block length with range. Similar to PTA, adaptive approaches for selecting the block size can be implemented. Also, some overlap between blocks should be considered to reduce the appearance of sidelobes due to phase jumps in the phase history of targets.

\section{Frequency Division (FD) Algorithm}

An algorithm based on multiple look processing was first presented in [5] to accommodate aperture-dependent motion errors, while in [6] a very similar algorithm (i.e., FD) was suggested, which also makes a comment on topography accommodation. It takes a similar approach as the SATA algorithm but makes the blocks (looks) in the frequency domain instead of in the time domain. Therefore, just before azimuth compression 
TABLE II

COMPUTATIONAL BURdEN OF THE ALGORITHMS UNDER STUDY WITH RESPECT TO FFT OPERATIONS

\begin{tabular}{lr}
\hline Algorithm & $O(\cdot)$ \\
\hline PTA & $\frac{N_{\mathrm{az}}}{1-\alpha} \cdot\left(\log _{2} N+N \cdot(1-\alpha)\right)$ \\
SATA & $\frac{N_{\mathrm{az}}}{1-\alpha} \cdot 2 \log _{2} N$ \\
FD & $\left(\frac{N_{\mathrm{az}}}{N}+1\right) \cdot N_{\mathrm{az}} \log _{2} N_{\mathrm{az}}$ \\
\hline
\end{tabular}

and after conventional second-order MoCo, data are Fourier transformed in the azimuthal direction. Afterward, only a small block in frequency (a look) with size $N$ is selected each time, which needs to be zero padded up to the original size of the data $N_{\text {az. }}$. The central frequency of this look corresponds to a certain squint, which is used to compute the appropriate correction. Therefore, after an inverse FT, a phase correction can be applied considering each time instant of the track deviations. Finally, a coherent addition of the different looks can be performed before the final azimuth compression step.

The performance of the FD is as follows. On one hand, the length of the look $N$ constrains the accommodation of the angle along the beam. Since the correction is applied considering the middle frequency in the look, a longer block will result in a worse angle accommodation. On the other hand, $N$ also defines the resolution in the accommodation of the topography, since the bandwidth of the look results in a certain synthetic aperture sublength. Hence, many small looks are preferred in the FD algorithm. The main advantage of FD is that it can accommodate track deviations accurately as the correction is applied at each time instant, although at the expense of computational burden. For every look, an inverse FT of $N_{\text {az }}$ points has to be performed, and the MoCo phase correction has to be applied for every look to the whole data matrix.

\section{Comments}

Each algorithm can be properly characterized using the formulas shown in Table I, namely the resolution in the accommodation of both the topography along azimuth $\delta x$ and the aperture angle $\delta \theta_{\mathrm{az}}$, and the maximum frequency of the residual error $f_{\max }^{\text {res }}$ with which the algorithm can cope after first- and second-order MoCo. These formulas have been obtained using basic SAR and signal processing relations. PRF is the effective pulse repetition frequency used during processing, $\theta_{\mathrm{az}}$ is the processed aperture beamwidth, $N$ is the number of azimuth samples per block, $L_{\mathrm{sa}}$ is the length of the synthetic aperture in seconds, $r$ is the closest approach range distance of the target, $\alpha(0 \leq \alpha<1)$ is an overlap factor, and $N_{\mathrm{az}}$ is the number of azimuth samples of the data matrix. Table II shows the computational burden considering only the operations related to the fast FT (FFT) for one azimuth line.

The formulas shown in Tables I and II are an excellent tool to analyze the performance of each algorithm. Fig. 1 plots these formulas for different values of the block size $N$. The system parameters in Table III have been used in this case, assuming a target at mid range. It is clear that PTA gives the best accommodation of topography, while FD can

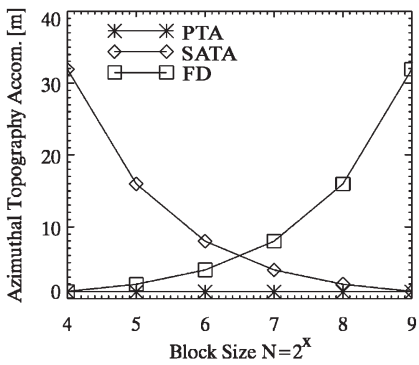

(a)

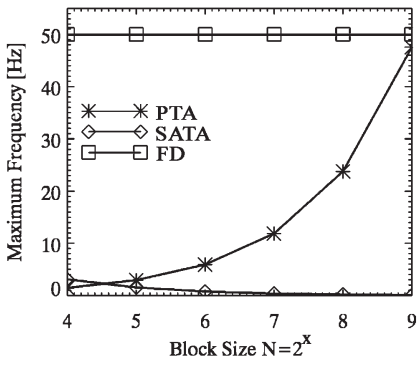

(c)

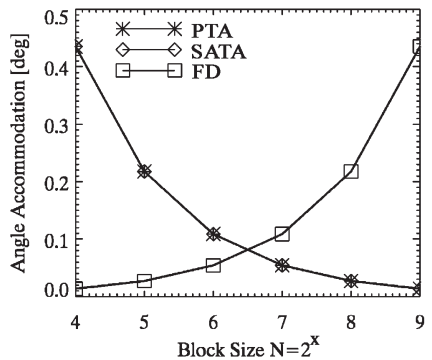

(b)

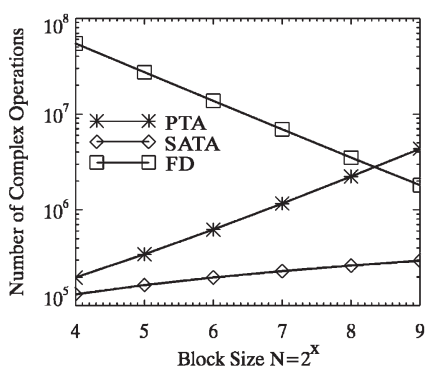

(d)
Fig. 1. Plots of the properties of the algorithms for different processing block sizes. (a) Topography accommodation accuracy $\delta x$. (b) Aperture angle accommodation accuracy $\delta \theta_{\mathrm{az}}$. (c) Maximum frequency of residual errors $f_{\max }^{\text {res }}$. (d) Computational burden for an azimuth line.

TABLE III

Main System AND Processing PARAMETERS

\begin{tabular}{lc}
\hline System $P R F$ & $400 \mathrm{~Hz}$ \\
Processed azimuth bandwidth & $100 \mathrm{~Hz}$ \\
Processed range bandwidth & $100 \mathrm{MHz}$ \\
Mid range & $4204 \mathrm{~m}$ \\
Mean flight altitude & $2600 \mathrm{~m}$ \\
Mean forward velocity & $95 \mathrm{~m} / \mathrm{s}$ \\
Azimuth samples $N_{\mathrm{az}}$ & 8192 \\
\hline
\end{tabular}

better accommodate fast track deviations. The performance of SATA seems to lie between these two, with a clear advantage concerning the computational burden. It is interesting to note that PTA is the only one that can achieve the best performance when the size of the block is equal to that of the synthetic aperture in samples ( $\left.N=L_{\mathrm{sa}} \cdot \mathrm{PRF}\right)$. Obviously, this will yield an important increase in computational burden. Nevertheless, all these algorithms focus on the azimuth signal, assuming RCMC has been properly carried out.

It should be noted that the requirements for fast track deviations are normally not very stringent, since the frequency of the deviations experienced by current airborne platforms is well under the limits shown in Table I, e.g., the maximum frequency of track deviations for the E-SAR system is $\sim 4 \mathrm{~Hz}$ [8]. Furthermore, the fact that first- and second-order MoCos have already been applied reduces even more the frequency of the needed correction. A fast variation where FD might perform better than the other two algorithms, e.g., several cycles of high amplitude within a synthetic aperture, is not realistic for current airborne platforms.

\section{Considerations in the Interferometric CASE}

It is of vital importance to understand the interferometric phase content of an interferometric pair after applying any 
of the approaches described above. In [9], it is shown that the flattened phase after applying MoCo assuming the same constant reference height for the whole scene is

$$
\phi_{\text {flat }}=-\frac{4 \pi}{\lambda} \frac{B_{\text {real }} \cos \left(\theta-\alpha_{\text {real }}\right)}{r_{t 0} \sin \theta} h=k_{z}^{\text {real }} \cdot h
$$

where $\theta$ is the off-nadir look angle, $r_{t 0}$ is the distance from the real antenna position, $B_{\text {real }}$ and $\alpha_{\text {real }}$ are the real baseline and tilt angle corresponding to the real trajectory, and $h$ is the height of the target with respect to the reference height assumed during MoCo. Equation (2) also assumes a constant deviation from the reference track along the synthetic aperture so that the values refer to the beam-center position.

Indeed, this is also the case if a DEM is used during MoCo. Assuming a perfect MoCo, the external DEM should be subtracted from the interferometric phase just in the same way as the flat earth is subtracted. Doing so, the residual phase is proportional to the DEM error $h_{\text {error }}$ and sensitive to the real baseline

$$
\phi_{\text {res }}(x, r)=k_{z}^{\text {real }}(x, r) \cdot h_{\text {error }}(x, r) .
$$

With this information, it is straightforward to correct the original DEM.

An important consideration in the case of using SATA or FD algorithms must be stated. Given that the topography accommodation accuracy is limited by the block size, the external DEM should be smoothed accordingly before using it to avoid wrong height estimates. This step will be necessary whenever the DEM resolution is better than that of the algorithms, where the latter is given by the values of $\delta x$ in Table I.

\section{RESUlts}

To compare the three approaches, L-band data acquired by the E-SAR system of DLR in a repeat-pass mode are used. The data have been processed with both ECS [1] and a time-domain backprojection algorithm. The latter is used for accuracy assessment as it applies topography- and aperture-dependent MoCo with no approximation. Hence, the accuracy of each algorithm can be estimated for a given scenario after comparison with the backprojection output. The data have been first processed using a Shuttle Radar Topography Mission (SRTM) DEM back geocoded to slant-range geometry. The spatial resolution of this DEM $(90 \times 90 \mathrm{~m})$ is low compared to the airborne image resolution. It is recommended to use efficient cubic convolution algorithms to obtain a smoothed interpolated DEM. Otherwise, SATA and FD will result in phase artifacts close to slope discontinuities [4]. The data have also been processed with a $5 \times 5 \mathrm{~m}$ resolution DEM obtained by the E-SAR at X-band in singlepass mode. Therefore, the accuracy in the accommodation of topography variations can be tested with both low- and highresolution DEMs. Table III summarizes the main system and processing parameters. The maximum horizontal and vertical deviations of the platform are \pm 6 and $\pm 5 \mathrm{~m}$, respectively.

Fig. 2 shows the reflectivity image and the SRTM DEM of the observed scene, where it can be noted that the scene has topographic variations of about $1000 \mathrm{~m}$. The output for each
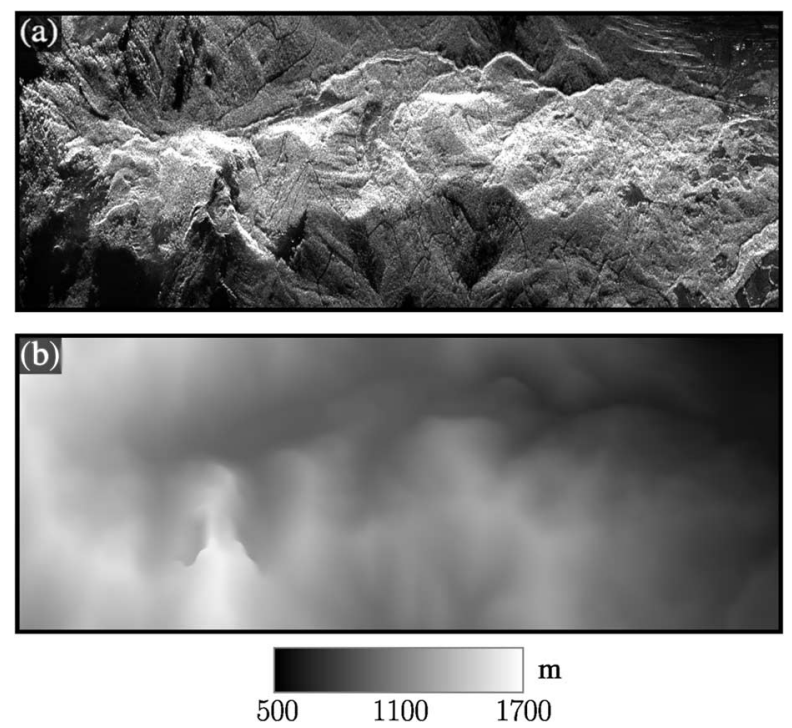

Fig. 2. (a) Reflectivity image and (b) back geocoded DEM in meters over the WGS-84 ellipsoid of the observed scene. Scene dimensions: $7 \times 2 \mathrm{~km}$ (azimuth $\times$ range). Near range on top of the image.
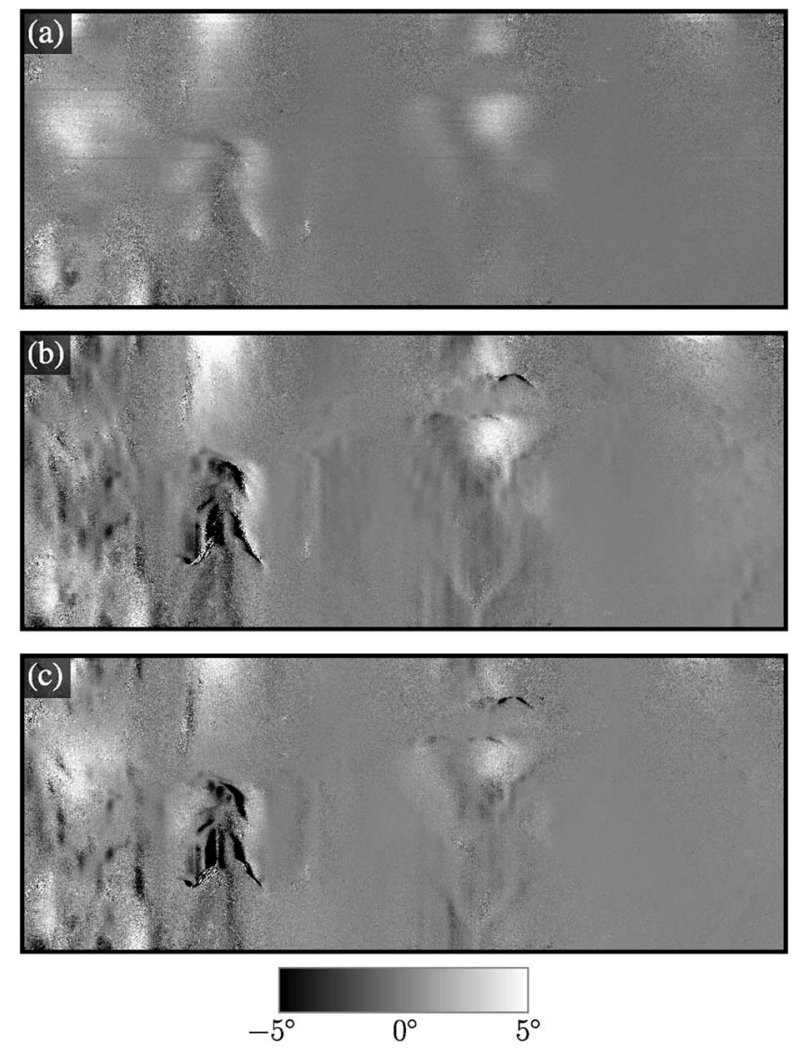

Fig. 3. Phase error $(N=32)$ for (a) PTA, (b) SATA, and (c) FD.

of the topography- and aperture-dependent algorithms has been compared with the output of the time-domain backprojection algorithm by means of a simple Hermitian product. Fig. 3 shows the phase error for each algorithm when using the SRTM DEM. It is clear that PTA has the best performance, as high topographic variations are accurately accommodated. On the other hand, both SATA and FD have problems to accommodate areas with high topographic changes; for example, in some places, the elevation changes are larger than $300 \mathrm{~m}$ in less 
TABLE IV

COMputational Burden AND ACCURACY

\begin{tabular}{lcccc}
\hline Algorithm & $\begin{array}{c}\text { Block Size } \\
(N)\end{array}$ & \multicolumn{2}{c}{ Phase error std. dev. } & Processing time \\
& SRTM & X-band & \\
\hline Time-domain & - & - & - & $4 \mathrm{~h} 25^{\prime} 30^{\prime \prime}$ \\
Conventional & - & $52.05^{\circ}$ & $39.02^{\circ}$ & $4^{\prime} 02^{\prime \prime}$ \\
\hline PTA & 32 & $1.08^{\circ}$ & $0.52^{\circ}$ & $19^{\prime} 32^{\prime \prime}$ \\
PTA & 64 & $1.08^{\circ}$ & $0.50^{\circ}$ & $18^{\prime} 15^{\prime \prime}$ \\
PTA & 128 & $1.07^{\circ}$ & $0.49^{\circ}$ & $28^{\prime} 29^{\prime \prime}$ \\
\hline SATA & 32 & $2.22^{\circ}$ & $2.34^{\circ}$ & $4^{\prime} 41^{\prime \prime}$ \\
SATA & 64 & $3.13^{\circ}$ & $3.83^{\circ}$ & $4^{\prime} 35^{\prime \prime}$ \\
SATA & 128 & $5.66^{\circ}$ & $6.39^{\circ}$ & $4^{\prime} 22^{\prime \prime}$ \\
\hline FD & 32 & $3.00^{\circ}$ & $3.05^{\circ}$ & $1 \mathrm{~h} 6^{\prime} 12^{\prime \prime}$ \\
FD & 64 & $3.01^{\circ}$ & $3.11^{\circ}$ & $35^{\prime} 58^{\prime \prime}$ \\
FD & 128 & $3.17^{\circ}$ & $3.28^{\circ}$ & $20^{\prime} 10^{\prime \prime}$ \\
\hline
\end{tabular}

than 40 azimuth samples. However, in general, the performance of all three algorithms can be considered excellent. Table IV shows the computational burden to process one image with each of the algorithms for different block sizes. The phase error standard deviation when compared to the time-domain processor is also shown for both SRTM and X-band DEMs. The result with conventional $\mathrm{MoCo}$, i.e., using the beam-center approximation and using the external DEM during second-order MoCo, is also included. Concerning the high-resolution X-band DEM, it is clear again that PTA shows the best performance, while SATA and FD fail to efficiently use the external information of the DEM.

Note that the computational burden decreases in some cases when the block size increases, which contradicts the formulas given in Table II. This is because Table II only considers the computational burden due to FFTs. However, other operations are carried out inside each block, e.g., true distance computation, complex products, etc., which have an important influence on the final processing time.

With regard to SATA, the error increases dramatically with the block size. As already commented, this is because the correction is applied considering the track position in the middle of the block. With a larger block, a bigger error is introduced at the edges, degrading in this way the result.

It is worth mentioning that the accuracy of the PTA does not vary significantly with an increasing block size, as shown in Table IV. This suggests that the residual errors to correct have low frequency components in this data set, so that even with a block size of 32 samples, they can be accommodated with PTA. Hence, increasing the block size does not perceptively improve the result in this case.

\section{CONCLUSION}

This letter has shown the comparison of different algorithms to accommodate topography- and aperture-dependent motion errors in airborne systems. These methods are thought to be implemented together with Fourier-based processors, such as ECS [1], range Doppler, or $\omega-k$ [10]. The need of such MoCo algorithms becomes evident in repeat-pass systems, where track deviations are not correlated, degrading in this way the retrieved interferogram.

After the theoretical and experimental results, it is possible to obtain clear conclusions. Concerning accuracy, PTA is the clos- est one to the ideal case. Indeed, with a block size equal to the synthetic aperture, it can accurately correct all phase errors. On the other hand, SATA shows the best accuracy-computational burden ratio. With a negligible computational cost, it achieves reasonable accuracy, which is acceptable in most scenarios with low to moderate topography. Note that SATA can result in large errors in airborne systems where fast track deviations can occur. In such cases, it is recommended to use PTA with a larger block size, or alternatively FD. Finally, FD results in a high computational burden when high accuracy is desired. The main advantage of this approach is that it can perfectly accommodate fast track variations. However, the computational effort is higher than for PTA for a similar topography accommodation resolution. Although only one data set is shown in this letter, similar performances have been obtained in other scenarios.

It has also been shown that the residual phase of an interferometric pair is proportional to the DEM error and the real baseline. Therefore, a smoothing of the DEM should be applied with SATA or FD whenever the DEM azimuthal resolution is better than that achievable by these algorithms.

Future work includes the optimization of the processing chain to reduce the errors that can be observed in the PTA case. Additionally, the comparison with fast time-domain processors, such as [11], will be a point to address in the future.

\section{ACKNOWLEDGMENT}

The authors would like to thank the anonymous reviewers for their valuable comments and suggestions.

\section{REFERENCES}

[1] A. Moreira, J. Mittermayer, and R. Scheiber, "Extended chirp scaling algorithm for air- and spaceborne SAR data processing in stripmap and scanSAR imaging modes," IEEE Trans. Geosci. Remote Sens., vol. 34, no. 5, pp. 1123-1136, Sep. 1996.

[2] G. Fornaro, G. Franceschetti, and S. Perna, "On center-beam approximation in SAR motion compensation," IEEE Trans. Geosci. Remote Sens., vol. 3, no. 2, pp. 276-280, Apr. 2006.

[3] K. A. C. de Macedo and R. Scheiber, "Precise topography- and aperturedependent motion compensation for airborne SAR," IEEE Trans. Geosci. Remote Sens., vol. 2, no. 2, pp. 172-176, Apr. 2005.

[4] P. Prats, A. Reigber, and J. J. Mallorqui, "Topography-dependent motion compensation for repeat-pass interferometric SAR systems," IEEE Trans. Geosci. Remote Sens., vol. 2, no. 2, pp. 206-210, Apr. 2005.

[5] R. Scheiber and V. Bothale, "Interferometric multi-look techniques for SAR data," in Proc. IEEE IGARSS, Toronto, ON, Canada, Jun. 24-28, 2002, vol. 1, pp. 173-175.

[6] X. Zheng, W. Yu, and Z. Li, "A novel algorithm for wide beam SAR motion compensation based on frequency division," in Proc. IGARSS, Denver, CO, Jul. 31-Aug. 4 2006, pp. 3160-3163.

[7] S. N. Madsen, "Motion compensation for ultra wide band SAR," in Proc. IGARSS, Sydney, Australia, Jul. 9-13, 2001, vol. 3, pp. 1436-1438.

[8] S. Buckreuss, "Motion errors in an airborne synthetic aperture radar system," Eur. Trans. Telecommun. Relat. Technol., vol. 2, no. 6, pp. 55-64, Nov. 1991.

[9] D. R. Stevens, I. G. Cumming, and A. L. Gray, "Options for airborne interferometric SAR motion compensation," IEEE Trans. Geosci. Remote Sens., vol. 33, no. 2, pp. 409-420, Mar. 1995.

[10] A. Reigber, E. Alivizatos, A. Potsis, and A. Moreira, "Extended wavenumber domain SAR focusing with integrated motion compensation," Proc. Inst. Electr. Eng._Radar Sonar Navig., vol. 153, no. 3, pp. 301310, Jun. 2006

[11] L. M. H. Ulander, H. Hellsten, and G. Stenstr, "Synthetic aperture radar processing using fast factorized back-projection," IEEE Trans. Aerosp. Electron. Syst., vol. 39, no. 3, pp. 760-776, Jul. 2003. 